\title{
Desempeño docente y satisfacción académica en estudiantes de educación secundaria
}

\section{Teaching performanceandacademicsatisfaction inhighschoolstudents}

\author{
Gesella Marlene Falcón Polanco \\ Universidad Nacional de Educación Enrique Guzmán y Valle
}

\section{Resumen}

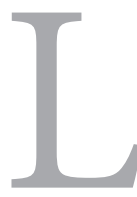

a presente investigación tuvo como objeto determinar la relación entre desempeño docente y satisfacción estudiantil. El trabajo presenta un diseño correlacional. La muestra estuvo conformada por 86 estudiante de la educación secundaria, los instrumentos utilizados fueron la encuesta para el desempeño docente elaborado y validado por juicio de expertos para esta investigación, consta de 10 ítems y tiene una escala de tipo Likert con una valoración de 1 a 4. Para evaluar satisfacción académica se utilizó la escala de Flores (2005) que consta de 30 ítems, dividida en 3 dimensiones: organización de la enseñanza, proceso de enseñanza aprendizaje, instalación e infraestructura la escala también es tipo Likert tiene una valoración de 1 a 4. A partir del análisis de los datos se concluye que existe relación significativa entre desempeño docente y satisfacción estudiantil.

\section{Palabras clave:}

Desempeño docente, satisfacción estudiantil, pedagogía de la docencia, organización de la enseñanza.

\section{Summary}

he objective of the present study was to determine the existing relationship between teaching performance and students' satisfaction. This work has a correlational design. The sample was composed by 86 high school students, the instruments used were the survey for teaching performance elaborated and validated through experts' judgement for this research. It consists of 10 items and it has a likert-type scale with a value scale from 1 to 4 . To evaluate academic satisfaction, we used the Flores scale (2005), which has 30 items, divided into 3 dimensions: teaching organization, teaching-learning process, and infrastructure and installations, which also have a likert-type scale with a value scale from 1 to 4 . From the result analysis we can conclude that there is a significant relationship between teaching performance and students' satisfaction.

\section{Key words:}

Teaching performance and students' satisfaction, teaching pedagogy, teaching organization. 


\section{Ciencia}

\section{Introducción}

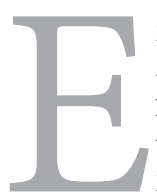

n los últimos años se ha hecho esfuerzos por parte del ministerio de educación de lograr cambios significativos en el sistema educativo, esto se orienta en la mayoría de los casos elevar el rendimiento académico del estudiante (Mendivil, 2011). El desempeño laboral califica la calidad profesional con la que se espera que haga el profesor de educación, mide variadas cualidades como pueden ser conductas en el trabajo, puntualidad, comportamiento, disposición para el trabajo, disciplina, compromiso institucional, desarrollo pedagógico, innovación etc (De la cruz, 2008).

Según Hernández y Sarramona (1986) el mejor modelo de desempeño docente es aquel que tiene en cuenta las características conceptuales referidas a lo que significa ser un buen docente o facilitador en un contexto determinado, ya que el concepto mismo de docente, es aquel que busca colaboración y superación de sus estudiantes con el propósito de lograr objetivos satisfactorios.

La razón de medir la satisfacción de los estudiantes, radica en el hecho de que son ellos el factor principal y garantía de la existencia y mantenimiento de las organizaciones educativas. Los estudiantes son los destinatarios de la educación, son los que mejor pueden valorarla y, aunque tienen una visión parcial, su opinión proporciona un referente que debe tomarse en cuenta (Gento y Vivas, 2003).
La satisfacción del estudiante refleja la eficiencia en diversos aspectos que componen experiencia educacional, estando relacionada con el rigor de los cursos, las interacciones con su profesor y los otros estudiantes, la justicia con que se le evalúa, así como con las instalaciones y equipamiento que apoyan dichas interacciones (Flores, 2005).

El concepto relacionado con "satisfacción estudiantil" se asocia al de "satisfacción del cliente", utilizado en la gestión de empresas; sin embargo, el término se matiza, toda vez que una universidad no es equiparable a una empresa, tampoco se debe confundir como clientes a los estudiantes puesto que son los destinatarios de la educación, no los clientes del mercado.

Los resultados abrirán un abanico de posibilidades, para mejorar el desempeño docente y puedan construir una propuesta de mejora en su desempeño laboral, buscando la satisfacción de los estudiantes y brindando aprendizajes significativos.

Del mismo modo, las autoridades podrán tomar decisiones adecuadas con la finalidad de mejorar el desempeño docente, en la medida que debe cumplirse eficientemente el accionar de los maestros en sus diversas áreas temáticas.

En este sentido la presente investigación tiene el propósito de establecer la relación entre desempeño docente y satisfacción estudiantil en el nivel secundario. El interés por el estudio es conocer cómo se desenvuelven los docentes en las aulas, considerando el marco de las buenas prácticas pedagógicas, propuesto por el ministerio de edu- 


\section{Ciencia}

cación del Perú (2007) frente a la satisfacción de los estudiantes, labor que desempeña el docente en el aula.

\section{Métodología}

Participantes. En este estudio participaron un total de 86 estudiantes de 13 y 14 años del segundo y tercer grado de educación secundaria,

Materiales. Se utilizó un cuestionario de desempeño docente que fue elaborado específicamente para esta investigación con el propósito de evaluar la precepción que tiene los estudiantes sobre el desempeño de sus profesores, el instrumento consta de 10 ítems y evalúa el factor de pedagogía de la docencia, contiene valoración de cuatro alternativas que va de (1) Nunca hasta (4) siempre. Para medir la satisfacción se utilizó una adaptación del cuestionario de satisfacción estudiantil de Flores (2005). Este cuestionario está dividido en 3 dimensiones: organización de la enseñanza, (10 ítems), proceso de enseñanza apren- dizaje (10 ítems), e instalación e infraestructura, (10 ítems) cada dimensión cuenta con 10 ítems, lo cual hace un total de 30 ítems, los estudiantes responderán a dicho cuestionario, en escala tipo Likert donde (1) es nunca y (4) es siempre.

Procedimiento. Para la aplicación del instrumento y obtener los datos se solicitó autorización a la directora de la institución educativa mediante un oficio. Luego de la aceptación se ingresó a las aulas y antes de aplicar los instrumentos se les presento las instrucciones a los estudiantes para resolver cualquier duda. y además se les presento el documento de consentimiento informado lo cual fue firmado por los estudiantes, posteriormente se le entrego los instrumentos para que resuelvan.

\section{Resultados}

A partir de los datos obtenidos de la aplicación de los instrumentos se analizaron los hallazgos obtenidos a nivel descriptivo e inferencial.

\begin{tabular}{lcccc}
\multicolumn{1}{c}{ Tabla 1 } & Desempeño docente. & \\
\hline & Frecuencia & Porcentaje & Porcentaje válido & Porcentaje acumulado \\
\hline A veces & 2 & 2 & 2 & 2 \\
\hline Casi siempre & 40 & 47 & 47 & 49 \\
\hline Siempre & 44 & 51 & 51 & 100 \\
\hline Total & 86 & 100 & 100 & \\
\hline
\end{tabular}

En la tabla 1 se observa que los estudiantes de educación secundaria presentan un nivel alto de percepción. Este resultado positivo por parte expertos, en una disciplina que aporta nuevas ideas al conocimiento de la realidad a través de su actividad.

del estudiante considera que los profesores son 


\section{Ciencia}

\begin{tabular}{lcccr}
\multicolumn{5}{c}{ Tabla 2 } \\
& Satisfacción académica. \\
\hline A veces & Frecuencia & Porcentaje & Porcentaje válido & Porcentaje acumulado \\
\hline Casi siempre & 8 & 9 & 9 & 9 \\
\hline Siempre & 54 & 63 & 63 & 72 \\
\hline Total & 24 & 28 & 28 & 100 \\
\hline
\end{tabular}

En la tabla 2: se observa un alto nivel de satisfacción académica en los estudiantes de la educación secundaria, los resultados positivos indican que los estudiantes adquieren cantidad de conocimientos, los docentes comprenden sus necesidades, son buenos guías para seguirlo.

Tabla 3

Correlación de variables

\begin{tabular}{|c|c|c|c|c|c|}
\hline & & $\begin{array}{l}\text { Satisfacción } \\
\text { académica. }\end{array}$ & $\begin{array}{l}\text { Organización de } \\
\text { la enseñanza }\end{array}$ & $\begin{array}{l}\text { Proceso de } \\
\text { enseñanza } \\
\text { aprendizaje }\end{array}$ & $\begin{array}{l}\text { Instalación e } \\
\text { infraestructura }\end{array}$ \\
\hline \multirow[t]{2}{*}{$\begin{array}{c}\text { Desempeño } \\
\text { docente }\end{array}$} & $\begin{array}{c}\text { Correlación de } \\
\text { Pearson }\end{array}$ & $.604\left({ }^{* *}\right)$ & $.669\left(^{* *}\right)$ & $.511\left({ }^{* *}\right)$ & $.334\left({ }^{* *}\right)$ \\
\hline & Sig.(bilateral) & 000 & 000 & 000 & 0.002 \\
\hline $\begin{array}{c}\text { Pedagogía de la } \\
\text { docencia }\end{array}$ & $\mathrm{N}$ & 86 & 86 & 86 & 86 \\
\hline
\end{tabular}

En la tabla 3 presentamos la correlación de variables entre desempeño docente, satisfacción académica y sus dimensiones existe una relación estadísticamente significativa, según el cuadro de Hernández Fernández y Baptista (2010) es correlación positiva baja de acuerdo a los índices de correlación. Discusión

\section{Discusión}

En el nivel descriptivo; la percepción sobre el desempeño docente, los estudiantes de educación secundaria presentan un nivel alto. Estos resultados positivos se deberían a que el concepto de profesor lo relacionan como el experto en una disciplina que aporta nuevas ideas al conocimiento de la realidad a través de su actividad como 


\section{Ciencia}

científico, pero con una formación pedagógica que le permite contribuir eficazmente a la formación de los estudiantes (Rocha, 2012). Cabalín et al. (2010), señala que una valoración positiva de la docencia pasa por lo que los docentes realizan en su centro de trabajo, tales como: realizar actividades relacionadas con la investigación, la creación y la validación del conocimiento.

Los estudiantes de educación secundaria, también presentan un nivel alto de satisfacción académica. Estos resultados están acordes con los resultados encontrados por Hernández, Lara, Ortega, Martínez y Avelino (2010) quienes proponen algunos indicadores por los cuales los estudiantes califican alto el desempeño de sus profesores, entre ellos tenemos: la cantidad de conocimientos adquiridos; el nivel de conocimientos adquiridos; el logro de objetivos; los trabajos realizados (todos ellos integrados en la variable proceso académico); la calidad de enseñanza impartida, competencia del profesorado (indicadores integrados a la variable docencia); las expectativas sobre desarrollo personal y profesional, las expectativas de cambio de estatus por estudiar una carrera, las expectativas por la aplicación de conocimientos en la vida profesional (de la variable desarrollo personal y profesional); la relación profesor-alumno (perteneciente a la variable relaciones interpersonales); la iniciativa personal en los trabajos realizados (variable implicación con la carrera); la percepción sobre las materias estudiadas, la percepción sobre la calidad de prácticas realizadas y la percepción sobre la cantidad de prácticas realizadas (indicadores integrados a la variable aspectos curriculares y administrativos).

A nivel inferencial, los resultados evidencian que existe una relación estadísticamente significativa entre la percepción del desempeño docente y satisfacción académica en estudiantes de educación secundaria. Estos resultados son acordes con los resultados encontrados en otros estudios similares (Bravo, 2010), y se debería principalmente a que existe coherencia entre lo que se enseña y la vida cotidiana de los estudiantes, la cual afecta no solo las relaciones con los maestros, sino, las relaciones que tiene cada estudiante con sus compañeros. Asimismo, estos resultados se deberían a lo que hace el maestro dentro del aula de clase y al material utilizado, según investigaciones previas, se ha comprobado que el material didáctico influye significativamente en el aprendizaje (Toribio, 2010). Además, Morocho (2010), llega a la conclusión que la metodología influye en el aprendizaje significativo de los estudiantes; por lo tanto, el uso de motivación, estrategias y medios materiales, favorece la producción de nuevos conocimientos mediante el desarrollo de capacidades, actitudes y sobre todo, la satisfacción de los estudiantes. En este sentido, haciendo referencia a lo que el docente hace, y como lo hace, Salinas y Martínez (2007) analizaron algunos de los aspectos que determinan la satisfacción del estudiante con la doble labor del profesorado: la docencia, por un lado y el diseño curricular de la 


\section{Ciencia}

asignatura, por el otro. Los resultados son coherentes en todas las dimensiones, dado que existe una relación significativa ente la metodología y el programa de estudio, confirmando una vez más que existente muchos factores que satisfacen académicamente al estudiante.

\section{Conclusiones}

A partir de los resultados encontrados se concluye que el desempeño docente frente a la satis-

\section{Referencias}

Bravo, J. (2010). Influencia de la motivación en el aprendizaje del curso de la física en los estudiantesde5toañodenivelsecundaria enlalnstituciónEducativaTúpacAmaru, ubicado en el cercado de Lima. (Tesis de Licenciatura). Universidad Federico Villareal, Perú.

Cabalín, D., et al. (2010). Concepción de estudiantes y docentes del buen profesor universitario. Facultad de medicina de la Universidad de La Frontera. International Journal of Morphology, 1(28), 283-290.

De la Cruz, L. (2007). Diccionario de educación. Lima -Perú. Recuperado de http://pucp-arte2009.blogspot.pe/2010/01/desempeno-docente-vs-rendimiento.html facción académica, se percibe que los estudiantes reciben información académica pertinente, los docentes dominan los saberes propios de su especialidad, tienen pericia pedagógica relacionada con aquello que enseña, Por ello, encontramos en esta investigación que existe relación significativa entre la percepción del desempeño docente y satisfacción académica en estudiantes de educación secundaria.

Flores, J. (2005). Encuesta sobre la Satisfacción Estudiantil. Revistaeléctrica deinvestigación docencia creativa 1, 29-37 Lima.

Gento, S. y Vivas, M. (2003). El SEUE: un instrumento para conocer la satisfacción de los estudiantes universitarios con su educación. Revista El Aula: Práctica y Reflexión. Recuperado de http://www.saber.ula.ve/db/ ssaber/Edocs/pubelectronicas/accionpedagogica/vol12num2/articulo_2.pdf.

Hernández, C.A., Lara, B., Ortega, M.P., Martínez, M.G. y Avelino, I. (2010). Evaluación de la satisfacción académica de los estudiantes de la licenciatura en didáctica del francés.RevistadeEducaciónyDesarrollo, Octubre-diciembre, 15, 35-46. Recuperado 


\section{Ciencia}

de http://www.cucs.udg.mx/revistas/edu_ desarrollo/anteriores/15/015_Hernandez. pdf.

Hernández, M. y Sarramona, J. (2002). La autoevaluación docente. Una propuesta para la formaciónpersonal. Revistaperfileseducativos (53-54), 58,61

Hernandez, R., Fernandez, C. y Baptista, P. (2014) Metodologia de la investigación. Mexico DF. McGraw-Hill.

Mendivil, L. (2011). ¿Qué aprender de las experiencias deevaluación docentequerealizamos. El caso peruano, Recuperado de http://riied.uniandes.edu.co/archivos/Seminarios/2_LuzmilaMendivilTrelles.pdf

Ministerio de Educación del Perú (2007). Sistema deevaluacióndedesempeñodocenteSEDD. Programa de educación en áreas rurales PEAR. Lima: MED, Gestión pedagógica.

Morocho, A. (2010). Influencia del aprendizaje significativo de las matemáticas, para el desarrollocognitivodelosalumnosdesecundarial.E.P."26dejunio"Independencia (Tesis de licenciatura). Universidad Federico Villarreal. Lima, Perú.

Rocha, R. (2012). La docencia universitaria desde la perspectiva de los alumnos frente a la de losprofesores.revistalnnovacionEducativa, enero-abril, 12(58), 91-118, Recuperado de file://C:/Users/Edson/Downloads/ Dialnet-LaDocenciaUniversitariaDesdeLaPersectivaDeLosAlum-4045913.pdf.

Salinas, A. y Martínez, P. (2007). Principales factores de satisfacción entre estudiantes universitarios. La unidad académica multidisciplinaria de Agronomía y Ciencias de la Universidad Autónoma de Tamaulipas, México. Sociotam, 17(1), 163-192.

Toribio, Y. (2010). Influencia del material didáctico en el aprendizaje de los alumnos del segundoañodelcolegioNuestraSeñorade Montserrat (Tesis de Licenciatura). Universidad Federico Villareal. Lima, Perú. 